\title{
QUASIMONOTONE EMBEDDING OF ONE-SIDED LIPSCHITZ CONTINUOUS FUNCTIONS
}

\author{
GERD HERZOG*
}

\begin{abstract}
We prove that one-sided Lipschitz continuous functions can be embedded into quasimonotone increasing functions, in a quite natural way. As a consequence of this result, quasimontonicity methods can be used to study initial and boundary value problems in Banach spaces if one-sided Lipschitz continuous functions are involved.
\end{abstract}

\section{Introduction}

Let $(E,\|\cdot\|)$ be a real Banach space, and let $m_{ \pm}: E \times E \rightarrow \mathrm{R}$ denote the one-sided directional derivatives of the norm, that is

$$
m_{ \pm}[x, y]:=\lim _{h \rightarrow 0 \pm} \frac{\|x+h y\|-\|x\|}{h} \quad(x, y \in E) .
$$

Let $D \subseteq E$. We call a function $g: D \rightarrow E$ one-sided Lipschitz continuous with constant $\mu \in \mathbf{R}$ if

$$
m_{+}[y-x, g(y)-g(x)] \leq \mu\|y-x\| \quad(x, y \in D),
$$

and if $I \subseteq \mathrm{R}$ is an interval, then $g: I \times D \rightarrow E$ is called one-sided Lipschitz continuous with function $\mu: I \rightarrow \mathrm{R}$, if $g(t, \cdot): D \rightarrow E$ is one-sided Lipschitz continuous with constant $\mu(t) \in \mathrm{R}$ for each $t \in I$.

Now, let $E$ be ordered by a cone $K$. A cone $K$ is a closed convex subset of $E$ with $\lambda K \subseteq K(\lambda \geq 0)$, and $K \cap(-K)=\{0\}$. As usual $x \leq y: \Longleftrightarrow$ $y-x \in K$, and we write $x \ll y$ if $y-x \in$ Int $K$. The subset

$$
K^{*}=\left\{\varphi \in E^{*}: \varphi(x) \geq 0 \quad(x \geq 0)\right\},
$$

of the space of all continuous linear functionals $E^{*}$, is called the dual wedge of $K$.

\footnotetext{
* The author is grateful to Prof. Dr. Roland Lemmert for discussions improving the paper.
}

Received February 15, 2006. 
A function $g: D \rightarrow E, D \subseteq E$ is quasimonotone increasing, in the sense of Volkmann [12], if

$$
x, y \in D, \quad x \leq y, \varphi \in K^{*}, \varphi(x)=\varphi(y) \Longrightarrow \varphi(g(x)) \leq \varphi(g(y)),
$$

and if $I \subseteq \mathrm{R}$ is an interval, then $g: I \times D \rightarrow E$ is called quasimonotone increasing if $g(t, \cdot): D \rightarrow E$ is quasimonotone increasing for each $t \in I$.

One-sided Lipschitz conditions are closely connected to a priori estimates, and to stability, invariance and existence results for dynamical systems and boundary value problems in Banach spaces, see for example [1], [2], [5], [8], [11], and in particular Martin's monograph [9], and the references given there. On the other hand, quasimonotone increasing functions are closely connected to differential inequalities, monotone dynamical systems, and boundary value problems in Banach spaces see for example [1], [3], [6], [7], [12], [13], [14] and the references given there.

In this paper we will prove that a one-sided Lipschitz continuous function can be embedded into a quasimonotone increasing function, in a quite natural way. As an application of this result, we will accomplish in some examples how results on one-sided Lipschitz continuous functions can be derived by results on quasimonotone increasing functions.

\section{A quasimonotonicity criterion}

Let $E$ be a Banach space ordered by a cone $K$. We assume that $K$ is reproducing, that is $K-K=E$, and that there exists $\Psi \in E^{*},\|\Psi\|=1$ such that

$$
\|x\|=\inf \{\Psi(p):-p \leq x \leq p\} \quad(x \in E) .
$$

Examples are $E=\mathrm{R}^{n}$ or $E=l^{1}(\mathrm{~N})$ with $K=\left\{x: x_{k} \geq 0\right\},\|x\|=$ $\sum_{k}\left|x_{k}\right|$, and $\Psi(x)=\sum_{k} x_{k}$. Note also that in some cases an equivalent norm can be defined by (1), for example in case $\operatorname{dim} E<\infty$ and if $\Psi \in K^{*}$ is such that $x \geq 0, \Psi(x)=0 \Rightarrow x=0$.

The following characterization of quasimonotone increasing functions can be found in [4].

Theorem 1. Let $D \subseteq E$, let $g: D \rightarrow E$ be a function, and let $\Psi$ be as above. Then the following assertions are equivalent:

(1) $g$ is quasimonotone increasing;

(2) $m_{+}[y-x, g(y)-g(x)]=\Psi(g(y)-g(x)) \quad(x, y \in D, x \leq y)$.

We reproduce the proof for reader's convenience.

Proof. We first prove

$$
K=\{x \in E: \Psi(x)=\|x\|\} .
$$


If $x \in K$ then obviously $\Psi(x)=\|x\|$. On the other hand, let $\Psi(x)=\|x\|$. To each $n \in \mathrm{N}$ there exists $p_{n} \in K$ such that

$$
\Psi\left(p_{n}\right) \leq\|x\|+\frac{1}{n}, \quad-p_{n} \leq x \leq p_{n} .
$$

Thus, $\left\|p_{n}-x\right\|=\Psi\left(p_{n}-x\right)=\Psi\left(p_{n}\right)-\|x\| \leq 1 / n$. Hence $x=\lim _{n \rightarrow \infty} p_{n} \geq$ 0 .

Next, we prove the following representation of $K^{*}$ : Let $\varphi \in E^{*} \backslash\{0\}$. Then

$$
\varphi \in K^{*} \Longleftrightarrow\left\|\Psi-\frac{\varphi}{\|\varphi\|}\right\| \leq 1
$$

Set $\chi=\Psi-\varphi /\|\varphi\|$. If $\|\chi\| \leq 1$ then

$$
\varphi(x)=\|\varphi\|(\|x\|-\chi(x)) \geq 0 \quad(x \in K),
$$

hence $\varphi \in K^{*}$. On the other hand, if $\varphi \in K^{*}$, then

$$
0 \leq \chi(x)=\|x\|-\frac{\varphi(x)}{\|\varphi\|} \leq\|x\| \quad(x \in K) .
$$

Fix $x \in E$, and let $\varepsilon>0$. Choose $p_{0}$ such that

$$
\Psi\left(p_{0}\right) \leq\|x\|+2 \varepsilon, \quad-p_{0} \leq x \leq p_{0} .
$$

Set

$$
x_{1}=\frac{p_{0}+x}{2}, \quad x_{2}=\frac{p_{0}-x}{2} .
$$

Then $x=x_{1}-x_{2}, x_{1}, x_{2} \in K$,

$$
\left\|x_{1}\right\|=\Psi\left(x_{1}\right)=\frac{1}{2}\left(\Psi(x)+\Psi\left(p_{0}\right)\right) \leq\|x\|+\varepsilon,
$$

and analogously $\left\|x_{2}\right\| \leq\|x\|+\varepsilon$.

Therefore

$$
-\|x\|-\varepsilon \leq-\left\|x_{2}\right\| \leq-\chi\left(x_{2}\right) \leq \chi\left(x_{1}-x_{2}\right) \leq \chi\left(x_{1}\right) \leq\left\|x_{1}\right\| \leq\|x\|+\varepsilon,
$$

that is $|\chi(x)| \leq\|x\|+\varepsilon$. For $\varepsilon \rightarrow 0+$ we obtain $|\chi(x)| \leq\|x\|$. Hence $\|\chi\| \leq 1$.

To prove the theorem we use Mazur's characterization of $m_{+}$, see [9], [10]:

$$
m_{+}[x, y]=\max \left\{\chi(y): \chi \in E^{*},\|\chi\|=1, \chi(x)=\|x\|\right\} .
$$

Let $g: D \rightarrow E$ be quasimonotone increasing, let $x, y \in D, x \leq y$, and let

$$
\chi \in E^{*}, \quad\|\chi\|=1, \quad \chi(y-x)=\|y-x\| .
$$


Then $\varphi:=\Psi-\chi \in K^{*}$, and

$$
\varphi(y-x)=\|y-x\|-\chi(y-x)=0 .
$$

Hence $\varphi(g(y)-g(x)) \geq 0$, that is

$$
\chi(g(y)-g(x)) \leq \Psi(g(y)-g(x)) .
$$

By means of (2) we have $m_{+}[y-x, g(y)-g(x)] \leq \Psi(g(y)-g(x))$. Equality follows from

$$
m_{+}[y-x, g(y)-g(x)] \geq \lim _{h \rightarrow 0+} \frac{\Psi(y-x+h(g(y)-g(x)))-\Psi(y-x)}{h},
$$

since $\|\Psi\|=1$.

Now, let $m_{+}[y-x, g(y)-g(x)] \leq \Psi(g(y)-g(x))$ be valid for $x, y \in D$, $x \leq y$.

Let $x, y \in D, x \leq y$, and $\varphi \in K^{*} \backslash\{0\}$ with $\varphi(x)=\varphi(y)$. For $\chi=$ $\Psi-\varphi /\|\varphi\|$ we know $\|\chi\| \leq 1$, and $\chi(y-x)=\|y-x\|$, in particular $\|\chi\|=1$. Equation (2) gives

$$
\chi(g(y)-g(x)) \leq m_{+}[y-x, g(y)-g(x)] \leq \Psi(g(y)-g(x)),
$$

that is

$$
\varphi(g(y)-g(x))=\|\varphi\|(\Psi-\chi)(g(y)-g(x)) \geq 0 .
$$

Hence $g$ is quasimonotone increasing.

Remark. From $m_{+}[x,-y]=-m_{-}[x, y](x, y \in E)$ we get: A function $g$ : $D \rightarrow E$ is quasimonotone decreasing (that is $-g$ is quasimonotone increasing) if and only if

$$
m_{-}[y-x, g(y)-g(x)]=\Psi(g(y)-g(x)) \quad(x, y \in D, x \leq y) .
$$

\section{Quasimonotone embedding}

In the sequel, let $(X,\|\cdot\|)$ be a real Banach space, and let the Banach space $Y=X \times \mathrm{R}$ be normed by $\|(x, \xi)\|=\max \{\|x\|,|\xi|\}$, and ordered by the cone

$$
K=\{(x, \xi) \in Y:\|x\| \leq \xi\} .
$$

The cone $K$ has nonempty interior, in fact $(x, \xi) \in \operatorname{Int} K$ if and only if $\|x\|<\xi$. In particular $K$ is reproducing and the dual wedge is a cone. Moreover $K$ is regular, that is each monotone and order bounded sequence is convergent. 
Theorem 2. Let $D \subseteq X$, let $f: D \rightarrow X$ be a function, let $\mu \in \mathrm{R}$, and let $F: D \times \mathbf{R} \rightarrow Y$ be defined by $F(x, \xi)=(f(x), \mu \xi)$. Then $f$ is onesided Lipschitz continuous with constant $\mu$ if and only if $F$ is quasimonotone increasing. Moreover if $f$ is one-sided Lipschitz continuous with constant $\mu$, then $F$ is one-sided Lipschitz continuous with constant $\mu$.

Proof. Consider $\Psi \in Y^{*}$ defined by $\Psi((x, \xi))=\xi$. Obviously $\|\Psi\|=1$. We prove that

$$
\|(x, \xi)\|=q((x, \xi)):=\inf \{\Psi((y, \eta)):-(y, \eta) \leq(x, \xi) \leq(y, \eta)\}
$$

for each $(x, \xi) \in Y$.

Let $-(y, \eta) \leq(x, \xi) \leq(y, \eta)$, that is

$$
\|y-x\| \leq \eta-\xi, \quad\|y+x\| \leq \eta+\xi .
$$

Thus $\eta \geq|\xi|$ and $2 \eta \geq\|y-x\|+\|y+x\| \geq 2\|x\|$, hence $\eta \geq\|(x, \xi)\|$. Therefore $q((x, \xi)) \geq\|(x, \xi)\|$.

On the other hand, if $\|x\| \leq|\xi|$ then $(x, \xi) \geq 0$ or $-(x, \xi) \geq 0$. In this case $q((x, \xi))=|\xi|=\|(x, \xi)\|$. If $\|x\|>|\xi|$, then an easy calculation shows

$$
-\left(\frac{\xi}{\|x\|} x,\|x\|\right) \leq(x, \xi) \leq\left(\frac{\xi}{\|x\|} x,\|x\|\right),
$$

and therefore $q((x, \xi)) \leq\|x\|=\|(x, \xi)\|$.

Next, note that

$$
\begin{aligned}
m_{+}[(x, \xi),(y, \eta)] & =\lim _{h \rightarrow 0+} \frac{1}{h}(\max \{\|x+h y\|,|\xi+h \eta|\}-\max \{\|x\|,|\xi|\}) \\
& = \begin{cases}m_{+}[x, y] & \text { if }\|x\|>|\xi|, \\
m_{+}[\xi, \eta] & \text { if }\|x\|<|\xi|, \\
\max \left\{m_{+}[x, y], m_{+}[\xi, \eta]\right\} & \text { if }\|x\|=|\xi| .\end{cases}
\end{aligned}
$$

Now, let $f$ be one-sided Lipschitz continuous with constant $\mu$, and let $(x, \xi) \leq$ $(y, \eta)$. Then $m_{+}[\eta-\xi, \mu(\eta-\xi)]=\mu(\eta-\xi)$. Thus, if $\|y-x\|<\eta-\xi$, then

$$
\begin{aligned}
m_{+}[(y, \eta)-(x, \xi), F((y, \eta))-F((x, \xi))] & =m_{+}[\eta-\xi, \mu(\eta-\xi)] \\
& =\mu(\eta-\xi) \\
& =\Psi(F((y, \eta))-F((x, \xi))),
\end{aligned}
$$


and if $\|y-x\|=\eta-\xi$, then $m_{+}[y-x, f(y)-f(x)] \leq \mu(\eta-\xi)$ and therefore

$$
\begin{aligned}
m_{+}[(y, \eta)-(x, \xi), F((y, \eta))-F((x, \xi))] \\
\quad=\max \left\{m_{+}[y-x, f(y)-f(x)], m_{+}[\eta-\xi, \mu(\eta-\xi)]\right\} \\
\quad=\mu(\eta-\xi) \\
\quad=\Psi(F((y, \eta))-F((x, \xi))) .
\end{aligned}
$$

According to Theorem $1 F$ is quasimonotone increasing.

If $F$ is quasimonotone increasing then by Theorem 1

$$
m_{+}[(y, \eta)-(x, \xi), F((y, \eta))-F((x, \xi))]=\mu(\eta-\xi),
$$

if $(x, \xi) \leq(y, \eta)$. Let $x, y \in X$ and choose $\xi, \eta \in \mathbf{R}$ such that $\|y-x\|=\eta-\xi$. Then

$$
\max \left\{m_{+}[y-x, f(y)-f(x)], m_{+}[\eta-\xi, \mu(\eta-\xi)]\right\}=\mu(\eta-\xi)
$$

and therefore

$$
m_{+}[y-x, f(y)-f(x)] \leq \mu(\eta-\xi)=\mu\|x-y\| .
$$

Finally, if $f$ is one-sided Lipschitz continuous with constant $\mu$, then

$$
m_{+}[(y, \eta)-(x, \xi), F((y, \eta))-F((x, \xi))] \leq \mu\|(y, \eta)-(x, \xi)\|
$$

for all $(y, \eta),(x, \xi) \in Y$ follows by the above representation of $m_{+}$in $Y$ by $m_{+}$in $X$ and $m_{+}$in R.

\section{Defect inequalities}

As a first application we consider Volkmann's monotonicity theorem [12, Satz 1], in our setting.

Let $D \subseteq X, T>0$, let $f:(0, T] \times D \rightarrow X$ be one-sided Lipschitz continuous with function $\mu:(0, T] \rightarrow \mathrm{R}$, and let $F:(0, T] \times(D \times \mathrm{R}) \rightarrow Y$ be defined by $F(t,(x, \xi))=(f(t, x), \mu(t) \xi)$. According to Theorem $2 F$ is quasimonotone increasing. Thus, if

$$
v=(x, \xi):[0, T] \rightarrow D \times \mathbf{R}, \quad w=(y, \eta):[0, T] \rightarrow D \times \mathbf{R}
$$

are continuous, differentiable on $(0, T]$ and such that

$$
v(0) \ll w(0), \quad v^{\prime}(t)-F(t, v(t)) \ll w^{\prime}(t)-F(t, w(t)) \quad(t \in(0, T])
$$


then $v(t) \ll w(t)(t \in[0, T])$. This means, that we have the following result on defect inequalities: If

$$
\|y(0)-x(0)\|<\eta(0)-\xi(0),
$$

and

$$
\begin{aligned}
\| y^{\prime}(t)-f(t, y(t)) & -\left(x^{\prime}(t)-f(t, x(t))\right) \| \\
& <\eta^{\prime}(t)-\mu(t) \eta(t)-\left(\xi^{\prime}(t)-\mu(t) \xi(t)\right) \quad(t \in(0, T]),
\end{aligned}
$$

then

$$
\|y(t)-x(t)\|<\eta(t)-\xi(t) \quad(t \in[0, T]) .
$$

Now, let $f:[0, T] \times X \rightarrow X$ be continuous and one-sided Lipschitz continuous with function $\mu \in C([0, T], \mathrm{R})$, and let $F:[0, T] \times Y \rightarrow Y$ be defined by $F(t,(x, \xi))=(f(t, x), \mu(t) \xi)$. Then $F$ is continuous, quasimonotone increasing, and moreover $F$ is one-sided Lipschitz continuous with the same function $\mu:[0, T] \rightarrow \mathrm{R}$. Since $\mu$ is bounded, the comparison theorem above holds for $C^{1}$-functions with $\ll$ replaced by $\leq$ everywhere, see [1, Theorem 5.4]. Thus we have proved:

Theorem 3. Let $f:[0, T] \times X \rightarrow X$ be continuous and one-sided Lipschitz continuous with function $\mu \in C([0, T], \mathbf{R})$, and let $(x, \xi),(y, \eta) \in$ $C^{1}([0, T], Y)$ be such that

$$
\|y(0)-x(0)\| \leq \eta(0)-\xi(0),
$$

and

$$
\begin{aligned}
\| y^{\prime}(t)-f(t, y(t)) & -\left(x^{\prime}(t)-f(t, x(t))\right) \| \\
& \leq \eta^{\prime}(t)-\mu(t) \eta(t)-\left(\xi^{\prime}(t)-\mu(t) \xi(t)\right) \quad(t \in[0, T]) .
\end{aligned}
$$

Then

$$
\|y(t)-x(t)\| \leq \eta(t)-\xi(t) \quad(t \in[0, T]) .
$$

In particular we can compare $y$ with a solution $x$ of $x^{\prime}(t)=f(t, x(t))$. By setting $\xi=0$ we obtain that $\|y(0)-x(0)\| \leq \eta(0)$ and $\left\|y^{\prime}(t)-f(t, y(t))\right\| \leq$ $\eta^{\prime}(t)-\mu(t) \eta(t)$ on $[0, T]$ imply $\|y(t)-x(t)\| \leq \eta(t)$ on $[0, T]$. In this form our result is in the spirit of the classical defect estimates for systems of differential equation, see [14, Chapter 11, Theorem 1].

\section{On Martin's existence theorem}

Let us say that a function $g:[0, T] \times E \rightarrow E$ is of at most linear growth, if $\|g(t, x)\| \leq a\|x\|+b((t, x) \in[0, T] \times E)$, for some constants $a, b \in \mathrm{R}$. To 
avoid technical difficulties we state Martin's existence Theorem [9, Chapter 6, Theorem 4.3], for such functions:

Theorem 4. Let $f:[0, T] \times X \rightarrow X$ be continuous, of at most linear growth, and one-sided Lipschitz continuous with function $\mu \in C([0, T], \mathrm{R})$. Then, the initial value problem

$$
x^{\prime}(t)=f(t, x(t)), \quad x(0)=x_{0}
$$

is uniquely solvable on $[0, T]$ for each $x_{0} \in X$.

We give here a proof of Theorem 4 by using the existence theorem of Lemmert, Schmidt and Volkmann [7]:

THEOREM 5. Let the Banach space $E$ be ordered by any regular cone with nonempty interior. Let $g:[0, T] \times E \rightarrow E$ be continuous, quasimonotone increasing and of at most linear growth. Then $u^{\prime}(t)=g(t, u(t)), u(0)=u_{0}$ is solvable on $[0, T]$ for each $u_{0} \in E$.

REMARK. In [7] this result is stated under the assumption that $g$ is bounded. Theorem 5 follows by minor changes in the original proof.

Proof of Theorem 4. First note that application of Theorem 3 proves that any two solutions of (3) coincide on their common domain of definition. Next, consider $F:[0, T] \times Y \rightarrow Y$ defined by $F(t,(x, \xi))=(f(t, x), \mu(t) \xi)$. Now, $F$ is continuous, quasimonotone increasing, and of at most linear growth:

$$
\|F(t,(x, \xi))\| \leq\left(a+\|\mu\|_{\infty}\right)\|(x, \xi)\|+b \quad((x, \xi) \in Y) .
$$

Since the cone $K$ is regular and has nonempty interior, Theorem 5 proves the existence of a solution $u=(x, \xi):[0, T] \rightarrow Y$ of the initial value problem

$$
u^{\prime}(t)=F(t, u(t)), \quad u(0)=\left(x_{0}, 0\right) .
$$

The associated function $x:[0, T] \rightarrow X$ is the solution of (3).

\section{Second order defect inequalities}

We say that $\mu:(0,1) \rightarrow \mathrm{R}$ has property $(P)$ if the second order differential inequality

$$
\lambda^{\prime \prime}(t)+\mu(t) \lambda(t)<0
$$

has a positive solution $\lambda:[0,1] \rightarrow(0, \infty)$, which is continuous and two times differentiable on $(0,1)$, compare [5].

Let $f:(0,1) \times X \rightarrow X$ be one-sided Lipschitz continuous with function $\mu$ having property $(P)$, and let $F:(0,1) \times Y \rightarrow Y$ be defined by $F(t,(x, \xi))=$ 
$(f(t, x), \mu(t) \xi)$. Then $F$ is quasimonotone increasing, and $F$ is one-sided Lipschitz continuous with the same function $\mu:(0,1) \rightarrow \mathrm{R}$. Thus we can apply [5, Theorem 3] on second order differential inequalities:

Let

$$
v=(x, \xi):[0,1] \rightarrow Y, \quad w=(y, \eta):[0,1] \rightarrow Y
$$

be continuous, two times differentiable on $(0,1)$, and such that

$$
\begin{aligned}
& v(0) \leq w(0), \quad v(1) \leq w(1), \\
& \quad v^{\prime \prime}(t)+F(t, v(t)) \geq w^{\prime \prime}(t)+F(t, w(t)) \quad(t \in(0,1)) .
\end{aligned}
$$

Then $v(t) \leq w(t)(t \in[0,1])$.

Transcription to a result on defect inequalities reads:

Theorem 6. Let $f:(0,1) \times X \rightarrow X$ be one-sided Lipschitz continuous with function $\mu:(0,1) \rightarrow \mathrm{R}$ having property $(P)$, and let $(x, \xi),(y, \eta):$ $[0,1] \rightarrow Y$ be continuous and two times differentiable on $(0,1)$ such that

$$
\|y(0)-x(0)\| \leq \eta(0)-\xi(0), \quad\|y(1)-x(1)\| \leq \eta(1)-\xi(1)
$$

and

$$
\begin{aligned}
\| x^{\prime \prime}(t)+f(t, x(t)) & -\left(y^{\prime \prime}(t)+f(t, y(t))\right) \| \\
& \leq \xi^{\prime \prime}(t)+\mu(t) \xi(t)-\left(\eta^{\prime \prime}(t)+\mu(t) \eta(t)\right) \quad(t \in(0,1)) .
\end{aligned}
$$

Then

$$
\|y(t)-x(t)\| \leq \eta(t)-\xi(t) \quad(t \in[0,1]) .
$$

REMARK. In particular, under the assumptions of Theorem 6 the boundary value problem $x^{\prime \prime}(t)+f(t, x(t))=0, x(0)=x_{0}, x(1)=x_{1}$ has at most one solution in $C([0,1], X) \cap C^{2}((0,1), X)$ for each choice of $x_{0}, x_{1} \in X$.

\section{An existence Theorem for systems of BVPs}

Let us say that $\mu \in C([0,1], \mathrm{R})$ has property $\left(P^{\prime}\right)$ if

$$
\lambda^{\prime \prime}(t)+\mu(t) \lambda(t) \leq 0
$$

has a positive solution $\lambda \in C^{2}([0,1],(0, \infty))$. For example $\mu$ has property $\left(P^{\prime}\right)$ if $\mu(t) \leq \pi^{2}$ on $[0,1]$ and $\mu \neq \pi^{2}$, but there are functions having property $\left(P^{\prime}\right)$ with even arbitrarily large maximum, see [2] and the references given there.

We have $\left(P^{\prime}\right) \Rightarrow(P)$, and the following existence theorem [2, Theorem 1] for the finite dimensional case is valid. 
THEOREM 7. Let the finite dimensional Banach space $E$ be ordered by a cone with nonempty interior, and let $g:[0,1] \times E \rightarrow E$ be continuous, quasimonotone increasing, and one-sided Lipschitz continuous with function $\mu \in C([0,1], \mathrm{R})$ having property $\left(P^{\prime}\right)$. Then $u^{\prime \prime}(t)+g(t, u(t))=0, u(0)=u_{0}$, $u(1)=u_{1}$ has a unique solution in $C^{2}([0,1], E)$ for each choice of $u_{0}, u_{1} \in E$, and the solution depends increasingly on $u_{0}$ and $u_{1}$.

Now, by means of Theorem 2, Theorem 7 implies the same assertion without the assumption of quasimonotonicity:

Theorem 8. Let $\operatorname{dim} X<\infty$, and let $f:[0,1] \times X \rightarrow X$ be continuous and one-sided Lipschitz continuous with function $\mu \in C([0,1], \mathrm{R})$ having property $\left(P^{\prime}\right)$. Then

$$
x^{\prime \prime}(t)+f(t, x(t))=0, \quad x(0)=x_{0}, \quad x(1)=x_{1}
$$

has a unique solution in $C^{2}([0,1], X)$ for each choice of $x_{0}, x_{1} \in X$. Moreover if $y \in C^{2}([0,1], X)$ is the solution of $y^{\prime \prime}(t)+f(t, y(t))=0, y(0)=y_{0}$, $y(1)=y_{1}$, and if $\delta \in C^{2}([0,1], \mathbf{R})$ is the solution of $\delta^{\prime \prime}+\mu(t) \delta(t)=0$, $\delta(0)=\left\|y_{0}-x_{0}\right\|, \delta(1)=\left\|y_{1}-x_{1}\right\|$, then

$$
\|y(t)-x(t)\| \leq \delta(t) \quad(t \in[0,1]) .
$$

Proof. Let $F:[0,1] \times Y \rightarrow Y$ be defined by $F(t,(x, \xi))=(f(t, x)$, $\mu(t) \xi)$. Now, $F$ is continuous, quasimonotone increasing, and one-sided Lipschitz continuous with the same function $\mu \in C([0,1], \mathrm{R})$. By Theorem 7 the boundary value problem

$$
u^{\prime \prime}(t)+F(t, u(t))=0, \quad u(0)=\left(x_{0}, 0\right), \quad u(1)=\left(x_{1}, 0\right)
$$

has a unique solution $u=(x, \xi) \in C^{2}([0,1], Y)$. The associated function $x:[0,1] \rightarrow X$ is the unique solution of (4). Finally, (5) follows by comparing $u$ with the solution $v=(y, \delta)$ of

$v^{\prime \prime}(t)+F(t, v(t))=0, \quad v(0)=\left(y_{0},\left\|y_{0}-x_{0}\right\|\right), \quad v(1)=\left(y_{1},\left\|y_{1}-x_{1}\right\|\right)$.

Remarks. The proof of Theorem 7 in [2] uses an existence theorem of Nagumo type (upper and lower solutions) from [3]. Mawhin [11, Chapter V] obtained results related to Theorem 8 for inner product spaces by degree methods. 


\section{REFERENCES}

1. Deimling, K., Ordinary Differential Equations in Banach Spaces, Lecture Notes in Math. 596 (1977).

2. Herzog, G., One-sided estimates for quasimonotone systems of boundary value problems, Demonstratio Math. 37 (2004), 847-856.

3. Herzog, G., The Dirichlet problem for quasimonotone systems of second order equations, Rocky Mountain J. Math. 34 (2004), 195-204.

4. Herzog, G., A characterization of quasimonotone increasing functions, Seminar LV, No. 19, 4 pp., 2004, http: / /www. mathematik. uni-karlsruhe.de/user/ Seminar_LV/

5. Herzog, G., Lemmert, R., Second order differential inequalities in Banach spaces, Ann. Polon. Math. 77 (2001), 69-78.

6. Hirsch, M. W., Smith, H., Monotone dynamical systems, Canada, A. (ed.) et al., Ordinary differential equations. Vol. II. Amsterdam, Elsevier/North Holland, Handbook of Differential Equations, 34c12, 2005.

7. Lemmert, R., Schmidt, S., Volkmann, P., Ein Existenzsatz für gewöhnliche Differentialgleichungen mit quasimonoton wachsender rechter Seite, Math. Nachr. 153 (1991), 349352.

8. Martin, R. H., Remarks on differential inequalities in Banach spaces, Proc. Amer. Math. Soc. 53 (1975), 65-71.

9. Martin, R. H., Nonlinear Operators and Differential Equations in Banach spaces, Robert E. Krieger Publ. Company, Malabar, 1987.

10. Mazur, S., Über konvexe Mengen in linearen normierten Räumen, Studia Math. 4 (1933), 70-84.

11. Mawhin, J., Topological degree methods in nonlinear boundary value problems, CBMS Regional Conf. Ser. in Math. No. 40, 1979.

12. Volkmann, P., Gewöhnliche Differentialungleichungen mit quasimonoton wachsenden Funktionen in topologischen Vektorräumen, Math. Z. 127 (1972), 157-164.

13. Volkmann, P., Gewöhnliche Differentialungleichungen mit quasimonoton wachsenden Funktionen in Banachräumen, Lecture Notes in Math. 415 (1974).

14. Walter, W., Differential and Integral Inequalities, Ergeb. Math. Grenzgeb. 55 (1970).

MATHEMATISCHES INSTITUT I

UNIVERSITÄT KARLSRUHE

D-76128 KARLSRUHE

GERMANY

E-mail: Gerd.Herzog@math.uni-karlsruhe.de 